\title{
Location-Oblivious Data Transfer with Flying Entangled Qudits
}

\author{
Adrian Kent ${ }^{1,2}$ \\ ${ }^{1}$ Centre for Quantum Information and Foundations, DAMTP, Centre for Mathematical Sciences, \\ University of Cambridge, Wilberforce Road, Cambridge, CB3 0WA, U.K. \\ ${ }^{2}$ Perimeter Institute for Theoretical Physics, 31 Caroline Street North, Waterloo, ON N2L 2Y5, Canada.
}

(Dated: October 30, 2018)

\begin{abstract}
We present a simple and practical quantum protocol involving two mistrustful agencies in Minkowski space, which allows Alice to transfer data to Bob at a spacetime location that neither can predict in advance. The location depends on both Alice's and Bob's actions. The protocol guarantees unconditionally to Alice that Bob learns the data at a randomly determined location; it guarantees to Bob that Alice will not learn the transfer location even after the protocol is complete.

The task implemented, transferring data at a space-time location that remains hidden from the transferrer, has no precise analogue in non-relativistic quantum cryptography. It illustrates further the scope for novel cryptographic applications of relativistic quantum theory.
\end{abstract}

\section{INTRODUCTION}

Because quantum states cannot be cloned, and can be entangled, someone who generates quantum states can retain more information about them than another party who receives them. Quantum cryptography exploits this information control (and other features of quantum theory) to implement interesting cryptographic tasks with security guaranteed so long as quantum theory is correct.

The no-signalling principle of special relativity also allows the controlled distribution of information, in the sense that a party can transfer classical or quantum information secure in the knowledge that it can only be communicated to the future light cone of the transfer point. The important cryptographic task of mistrustful coin tossing [1] can be implemented using this fact alone. More surprisingly, it can also be used to implement the (strictly more powerful [2] ) task of bit commitment, via protocols that require only classical information exchanges but which guarantee security against Mayers-Lo-Chau quantum attacks [3-7] as well as all classical attacks 9, 10.

We would like, for both theoretical and practical reasons, to understand precisely which cryptographic tasks can be implemented when we take relativistic quantum theory to be the underlying theory, and thus combine the information control techniques allowed by both theories. There have been several interesting recent illustrations [11, 12] of the cryptographic power of relativistic quantum theory. Work on quantum tagging and position-based quantum cryptography has also produced new cryptographic schemes for quantum tagging secure against technologically restricted adversaries $[13-$ 16], some possible limits to the level of security attainable for some versions of these tasks [15, 16] and an unconditionally secure protocol [17] for quantum tagging given a tagging device that is cryptographically secure - i.e. that can keep internal data secret from an adversary. Intriguing extensions of quantum tagging to more general position-based cryptographic tasks have also been developed [15].
Light speed constraints are already practically significant in terrestrial technology, even on small scales, and will become far more so as we and our technology migrate further beyond the Earth. So it seems natural not only to try to apply these constraints to implement classical (non-quantum non-relativistic) tasks, but also to rethink what cryptography actually means - what the most general interesting and potentially useful cryptographic tasks are - in a world governed by relativity and quantum theory. Although Shor's and Grover's algorithms, and other efficient quantum algorithms for intrinsically classical computational tasks, were seminal discoveries which added great impetus to the development of quantum computational theory, many believe that intrinsically quantum computational tasks may well ultimately be the most significant applications of quantum computing. It seems by now quite plausible too that intrinsically relativistic and quantum cryptographic tasks will also ultimately be among the most significant applications of physics-based cryptography and computing. At the very least, it seems worth exploring what might be possible with this vision in mind.

In this paper we define a new and intrinsically relativistic cryptographic task, data transfer at a spacetime location that remains hidden from the transferring party. We give a simple and practical protocol for implementing this task, and show the protocol is unconditionally secure, in an appropriately defined sense. The protocol is inspired by the no-summoning theorem [18] and its application to bit commitment [12]. Roughly speaking, the underlying intuition is that, by producing one qudit of an entangled pair at an unpredictable point, Alice effectively constrains Bob, who has no way of ensuring that he can produce the other qudit at or near the same point. However, the security proof goes beyond the nosummoning theorem, using other features of relativistic quantum theory. In particular, it implicitly assumes that the unitary evolution of quantum states between any two spacelike hypersurfaces can be covariantly defined, as in the Tomonaga-Schwinger formalism [19, 20]. 


\section{TRANSFERRING DATA WITH ENTANGLED FLYING QUDITS}

We give an idealized version of the protocol here to simplify the presentation. ${ }^{1}$ We suppose that space-time is Minkowski and that nature is described by some appropriate relativistic version of quantum theory. We consider two agencies, Alice and Bob, with representatives at all the relevant points in space-time, and suppose that both have arbitrarily efficient technology, limited only by physical principles, and that all their operations and communications are error-free. We also suppose they agree in advance on some space-time point $P$, where the protocol commences, and on a fixed inertial reference frame.

We suppose they are also independently able to access suitable points $Q_{i}$ (where $i$ runs from 0 to $m-1$ ) in the causal future of $P$, and that each is able to keep information everywhere secure from the other unless and until they choose to disclose it. We also suppose that Alice and Bob can independently and securely access all the points $P$ and $Q_{i}$ and instantaneously exchange information there. In particular, Alice can keep a bipartite entangled state private somewhere in the past of $P$ and arrange to transfer one subsystem to Bob at $P$, and Alice's and Bob's subsystems can then be kept private unless and until they choose to transfer them at some point(s) in the future of $P$. We also suppose that both parties can send any relevant states at light speed in prescribed directions along secure quantum channels.

Apart from our idealized assumptions about independent access to suitable space-time points, we are following here the standard conventions of mistrustful cryptography. Alice and Bob mistrust one another, and each trusts only operations they carry out themselves in their own secure "laboratories". As is standard in mistrustful cryptography, the aim of our protocol is to offer each some guaranteed control over the information obtained by the other, despite their mistrust. We need not consider the actions of any third parties (for instance an interfering eavesdropper Eve), since as far as Alice is concerned everything outside her laboratory is potentially under Bob's control, and vice versa.

We focus here on a specific version of the protocol, with $m=2$ : the protocol can obviously be extended to larger $m$, to various geometries, and to variants involving multipartite entanglement. For $m=2$, Alice and Bob agree two distinct spatial directions $v_{0}$ and $v_{1}$ in the agreed frame. For simplicity, we focus here on one natural choice, in which the $v_{i}$ are opposite; we set $c=1$, take $P$ to be the origin and the two spatial directions to be de-

\footnotetext{
${ }^{1}$ As in other applications [12] inspired by the no-summoning theorem [18], these unphysical idealizations can be relaxed without affecting the essential idea, and it is easy to adjust the protocol so that it can be securely implemented under realistic physical assumptions, allowing for noise and time lags. See Refs. [12, 18] for illustrations.
}

fined by the vectors $v_{0}=(-1,0,0,0)$ and $v_{1}=(1,0,0,0)$ in the fixed frame coordinates $(x, y, z, t)$.

Before the protocol, Alice generates a two qudit state; the dimension $d$ is a pre-agreed parameter. This state determines the datum that will be transferred in the protocol. If the protocol is followed, Bob will receive an integer in the range $1, \ldots, d^{2}$. Suppose for the moment that Alice wishes this integer to be classically determined from the start of the protocol. She then prepares a maximally entangled state $\psi_{i} \in \mathcal{C}_{A}^{d} \otimes \mathcal{C}_{B}^{d}$, chosen from a preagreed orthonormal basis labelled by $i=1, \ldots, d^{2}$. This is encoded in two physical subsystems which (idealizing again) we take to be pointlike. She keeps the state private until $P$, where she gives the second subsystem to Bob. Alice now uses a private equiprobable random bit $j \in\{0,1\}$ and sends the first subsystem along a secure channel that she controls at light speed in the direction $v_{j}$, i.e. along the line $L_{0}=\{(-t, 0,0, t), t>0\}$ (for $j=0$ ) or the line $L_{1}=\{(t, 0,0, t), t>0\}$ (for $\left.j=1\right) .{ }^{2}$

Bob is free to act as he wishes, depending on his preferred distribution for the point of data transfer. If he wishes to maximize his chance of obtaining the data at the earliest ${ }^{3}$ possible point in space-time, then he also randomly chooses a bit $k \in\{0,1\}$ by flipping a fair coin and sends the second subsystem along a secure channel that he controls at light speed in the direction $v_{k}$.

To transfer the datum to Bob, Alice gives him the second subsystem at some point $Q_{j}$ along the line $L_{j}$. The points $Q_{0}$ and $Q_{1}$ (but not Alice's choice $j$ ) could be agreed in advance, in which case Bob knows to expect the data at one of two possible points. However, if Alice wishes to retain freedom in choosing the transfer point, she can choose any point $Q_{j}$ along $L_{j}$, provided that Bob is prepared to receive data there. To simplify here, we assume $Q_{0}=\{-T, 0,0, T\}$ and $Q_{1}=\{T, 0,0, T\}$ are fixed, for some agreed choice of $T$.

The transferred datum is the value of $i$. To obtain this information, Bob needs to carry out a measurement of the two subsystems in the agreed orthonormal basis. His measurement need not necessarily be local; however, if not, then Bob will possess the datum $i$ only at points where he is able to combine all the data obtained from non-local measurement operations.

\section{A. Security against Alice}

Alice has little scope for cheating. She can choose the joint state of the two subsystems to be a state other than one of the basis states $\psi_{i}$. If she does, Bob will ultimately

\footnotetext{
2 By requiring light speed transmission in opposite directions we ensure the possible data transfer points separate as fast as possible in the given frame. Neither constraint is necessary, however: the protocol achieves location-oblivious data transfer so long as $Q_{0}$ and $Q_{1}$ are spacelike separated.

3 With respect to the Minkowski causal partial ordering.
} 
obtain an $i$ corresponding to the random outcome of his measurement. Nonetheless, Bob obtains a value of $i$. The aim of this protocol is simply to ensure that Alice transfers some datum to Bob: as with classical oblivious transfer [8], there is no requirement that the datum transferred should be "correct" by any external standard. Nor do we require that the datum be classically determined before the protocol is complete - something which is not possible with any intrinsically quantum protocol (see e.g. [21]). Alice's freedom in choosing the joint state thus does not constitute cheating.

Alice's only other freedom is to choose $j$ by some procedure other than flipping a fair coin. This gives her no advantage, assuming - as we will here - that Alice and Bob each assign equal utility to Bob knowing the datum at points $(x, y, z, t)$ and $(-x, y, z, t)$, for any coordinate values. ${ }^{4}$

Moreover, given this symmetry of utilities, it can only help Bob if Alice deviates from unbiased randomness: if he learns some information about Alice's strategy for choosing $j$ he may alter his own strategy in response, and thus increase the chance that the datum is transferred to him earlier. We need not consider this possibility, since it involves both parties violating the protocol ${ }^{5}$, but in any case there is no motivation for Alice to pursue this strategy.

\section{B. Security against Bob}

Bob may act as he wishes, constrained only by the laws of quantum theory and relativity. By definition, he cannot cheat. Interestingly, though, he is nonetheless quite constrained. We say Bob learns ${ }^{6}$ the correct value of $i$ if he obtains it by some strategy which (in the ideal error-free case) guarantees that, if it produces an answer $i^{\prime}$ and Alice chose a classically pre-determined $i$, then $i=i^{\prime}$.

Suppose first that he follows the strategy above, sending his qudit in a randomly chosen direction $v_{k}(k=0$ or 1), and suppose without loss of generality that $k=0$. He then learns the value of $j$ at $Q_{0}$, where he either receives the second qudit $(j=0)$ or does not $(j=1)$; he similarly learns this value at $Q_{1}$. If $j=0$, he can obtain the datum $i$ at $Q_{0}$, by carrying out a local measurement on both qudits there. If $j=1$, he now has the two qudits at spacelike separated locations, $Q_{0}$ and $Q_{1}$, and is aware of the situation at both locations. For any point $X$, we write $L(X)$ for the set containing $X$ and its future

\footnotetext{
${ }^{4}$ If their utilities are asymmetric, things become more complicated, and we need game theory to determine Alice's and Bob's optimal actions.

5 As usual in mistrustful cryptography, the aim is to protect an honest party against a cheat; we do not aim to protect cheats against the consequences of their own misbehaviour.

${ }^{6}$ I.e. comes to know with certainty.
}

light cone. Bob can obtain the datum $i$ at any point $Y$ in the intersection $L\left(Q_{0}\right) \cap L\left(Q_{1}\right)$ by sending the two qudits from $Q_{0}$ and $Q_{1}$ to $Y{ }^{7}$ And this is the best Bob can do when he holds the two entangled qudits at $Q_{0}$ and $Q_{1}$ : he cannot then learn the datum $i$ at any location outside $L\left(Q_{0}\right) \cap L\left(Q_{1}\right)$.

In summary, following this strategy, with probability $\frac{1}{2}$ he learns $i$ at $Q_{j}$, and with probability $\frac{1}{2}$ he learns $i$ only at $Y$. Note that Alice never learns $k$, and so does not know the location at which Bob learns $i$, since all Bob's operations are private.

In fact, the above strategy is optimal for Bob, in the following sense: (i) Bob has no strategy that gives him probability greater than 0 of learning $i$ at any point outside $L\left(Q_{j}\right)$, and (ii) Bob has no strategy that gives him probability greater than $\frac{1}{2}$ of learning $i$ at any point outside $L\left(Q_{0}\right) \cap L\left(Q_{1}\right)$.

To see (i), we simply observe that Bob has no access to the subsystem defined by $\mathcal{C}_{A}^{d}$ until Alice transfers it at $Q_{j}$.

To see (ii), consider a spacelike hypersurface $S$ close to and in the past of the boundary of $L\left(Q_{0}\right) \cup L\left(Q_{1}\right)$. For simplicity, take $S$ to be symmetric under reflections in the $x$-coordinate, and define the subsurfaces $S_{0}=\{(x, y, z, t) \in S: x \leq 0\}$ and $S_{1}=\{(x, y, z, t) \in$ $S: x \geq 0\}$.

Without loss of generality, we can consider Bob's actions between $P$ and $S$ as defined by a unitary operation acting on $\mathcal{C}_{B}^{d}$ together perhaps with ancillary states, generating some quantum state on $S$. Hence at least one of the states defined by the restriction of this state to $S_{0}$ or $S_{1}$ must be entangled with the $\mathcal{C}_{A}^{d}$ state held by Alice. As Bob does not know $j$ until after the points $Q_{0}$ and $Q_{1}$, his actions up to $S$ are independent of the choice of $j$. Hence, with probability $\geq \frac{1}{2}$, Bob generates a state on $S_{\bar{j}}$ - the opposite half of the hypersurface to $Q_{j}$ - that is entangled with the state transferred by Alice at $Q_{j}$. Bob thus cannot learn the outcome of the desired measurement at any point not in the intersection of the future light cone of $Q_{j}$ and the future light cone of at least one point in $S_{\bar{j}}$. As we can find spacelike $S$ arbitrarily close to the boundary of $L\left(Q_{0}\right) \cup L\left(Q_{1}\right)$, the result follows.

\section{DISCUSSION}

The task defined here, transferring data at a location that remains hidden from the transferrer, has no precise analogue in classical or non-relativistic quantum cryptography, although it bears some resemblance to classical oblivious transfer [8]. Like classical oblivious transfer,

7 He could also achieve this by a variety of other strategies, for example by using a second predistributed entangled pair of qudits to carry out a nonlocal measurement at $Q_{0}$ and $Q_{1}$ and sending the results to $Y$. 
it is probably not very useful in itself, but could be a building block for much more useful tasks (as oblivious transfer is [22]).

In any case, it illustrates some interesting possibilities in relativity-based cryptography. First, one can aim to control where exactly in spacetime various parties learn information as a result of a protocol. In our protocol this control is randomized; it would also be interesting to explore the (im)possibility of deterministic location control. Second, the locations at which information is learned themselves constitute information generated by the protocol, and the flow of this information is also controlled. Moreover, this control can be asymmetric - in our protocol, Bob learns the location information, and Alice never does.

These possibilities raise some intriguing questions. For example, one can imagine secure multi-party computation [23] scenarios in which parties are supposed to learn some outputs given by prescribed joint functions of the inputs, but it does not matter that various parties eventually learn partial or even complete data about other parties' input data, as long as there are strong enough constraints on where in spacetime they learn this. It would be very interesting to understand whether, and under what conditions, such constraints can be implemented securely.

Our protocol imposes strong constraints on Bob whatever it does. However, it should be stressed that he has more general possibilities than those highlighted above, if he is willing to accept some risk that he will never obtain the correct value of Alice's input datum. For example, he could carry out some form of partial cloning on his qudit and send the two outputs to $Q_{0}$ and $Q_{1}$ respectively, and then obtain some information about $i$ by a local measurement at $Q_{j}$, whichever $j$ Alice chooses. It would be interesting to characterize the tradeoffs available to Bob via his most general strategies.

Finally, one might perhaps wonder whether the protocol is intrinsically quantum, or whether it could perhaps be implemented classically. For example, could Alice initially give Bob a classical bit $b_{0}$ at $P$, and a second bit $b_{1}$ at one of the $Q_{i}$, in order to transfer the bit sum $b=b_{0} \oplus b_{1}$ ? A little thought shows this does not restrict Bob, since once he receives $b_{0}$ he can simply broadcast it in all directions, ensuring that he obtains $b$ at whichever point Alice chooses to return $b_{1}$. More generally, since the security proof relies on the no-summoning theorem, which holds in relativistic quantum theory but not in relativistic classical theories, no classical protocol can achieve the same result.

\section{Acknowledgments}

This work was partially supported by an FQXi minigrant and by Perimeter Institute for Theoretical Physics. Research at Perimeter Institute is supported by the Government of Canada through Industry Canada and by the Province of Ontario through the Ministry of Research and Innovation.
[1] M. Blum, in Proceedings of the 24th IEEE Computer Conference ( Compcon), pp. 133-137 (IEEE, New York,1982).

[2] A. Kent, Coin Tossing is Strictly Weaker Than Bit Commitment, Phys. Rev. Lett. 83 (1999) 5382-5384.

[3] D. Mayers, Unconditionally secure quantum bit commitment is impossible, Phys. Rev. Lett. 78 3414-3417 (1997).

[4] D. Mayers, The trouble with quantum bit commitment, quant-ph/9603015 3 .

[5] D. Mayers, Unconditionally secure quantum bit commitment is impossible, Proceedings of the Fourth Workshop on Physics and Computation (New England Complex System Inst., Boston, 1996), p. 226.

[6] H.-K. Lo and H. Chau, Is quantum bit commitment really possible?, Phys. Rev. Lett. 78 3410-3413 (1997).

[7] H.-K. Lo and H. Chau, Why quantum bit commitment and ideal quantum coin tossing are impossible, Proceedings of the Fourth Workshop on Physics and Computation (New England Complex System Inst., Boston, 1996), p. 76 .

[8] Michael O. Rabin. "How to exchange secrets by oblivious transfer." Technical Report TR-81, Aiken Computation Laboratory, Harvard University, 1981

[9] A. Kent, Unconditionally secure bit commitment, Phys. Rev. Lett. 83 1447-1450 (1999).
[10] A. Kent, Secure Classical Bit Commitment using Fixed Capacity Communication Channels, J. Cryptology 18 (2005) 313-335.

[11] R. Colbeck and A. Kent, Variable Bias Coin Tossing, Phys. Rev. A 73, 032320 (2006).

[12] A. Kent, Unconditionally Secure Bit Commitment with Flying Qudits, arXiv:1101.4620 (2011).

[13] A. Kent, R. Beausoleil, W. Munro and T. Spiller, Tagging Systems, US patent US20067075438 (2006).

[14] R. Malaney, Phys. Rev. A 81, 042319 (2010); arXiv:1004.4689 (2010).

[15] N. Chandran et al., arXiv:1005.1750 (2010).

[16] A. Kent, W. Munro and T. Spiller, Quantum Tagging: Authenticating Location via Quantum Information and Relativistic Signalling Constraints, arXiv:1008.2147 (2010), to appear in Phys. Rev. A.

[17] A. Kent, Quantum Tagging with Cryptographically Secure Tags, arXiv:1008.5380 (2010).

[18] A. Kent, A No-summoning theorem in Relativistic Quantum Theory, arXiv:1101.4612 (2011).

[19] S. Tomonaga, On a Relativistically Invariant Formulation of the Quantum Theory of Wave Fields, Prog. Theor. Phys. 1 (1946) 27.

[20] J. Schwinger, Quantum Electrodynamics, I - A covariant formulation, Phys. Rev. 74 (1948) 1439. 
[21] A. Kent,Why Classical Certification is Impossible in a Quantum World, quant-ph/0409029, to appear in Quantum Information Processing.

[22] J. Kilian, Founding Cryptography on Oblivious Transfer, Proceedings of the 20th Annual ACM Symposium on the Theory of Computation, Chicago, 1988 (ACM,
New York, NY, 1988).

[23] A. Yao, Protocols for Secure Computations (Extended Abstract), in Proceedings of the 25th Annual ACM Symposium on the Theory of Computation, Montreal, 1994 (ACM, New York, 1994) pp 160-164. 